\title{
Editorial: The Impact of Smart Screen Technologies and Accompanied Apps on Young Children Learning and Developmental Outcomes
}

\author{
Stamatios Papadakis ${ }^{1 *}$, Michail Kalogianakis ${ }^{1}$, Eirini Sifaki ${ }^{2}$ and Angeliki Monnier ${ }^{3}$ \\ ${ }^{1}$ Department of Preschool Education, University of Crete, Rethymno, Greece, ${ }^{2}$ Department of Social Sciences, Hellenic Open \\ University, Patras, Greece, ${ }^{3}$ UFR Sciences Humaines et Sociales, Université de Lorraine, Metz, France
}

Keywords: smart screen technologies, applications, young children, learning, development

Editorial on the Research Topic

The Impact of Smart Screen Technologies and Accompanied Apps on Young Children Learning and Developmental Outcomes

The first job of any journal editorial ought to be to celebrate the accomplishment of the contributors included. As you read the five articles in this present collection, we invite you to think of the degree of scholarship in our relationship with technology and young children.

Every collection of papers is fascinating in its way, and this collection is no exception. There are papers from countries as diverse as Greece, Russia, Turkey, and the United States. The topics reflect new issues that demand attention, such as the role of smart screen technologies and digital learning. At the same time, they include ongoing themes of inquiry on the understanding, beliefs, values, and differing models of educational relationship and the development of inclusive practice. The first article was a literature review that examined the impact of coding apps to support children's CT skills. The next two articles examined the use of technology and media in preschool classrooms. The last two articles studied the role of social robots and screens in vocabulary learning. We hope that the reader will find a helpful reference for the state-of-the-art in the emerging field of smart screen technologies on young children's learning and developmental outcomes.

The first article (Papadaki) presents a literature review of empirical studies on applying four coding apps to support young children's learning of Computational Thinking (CT) and Computational Fluency (CF). The main conclusion is that all apps positively affect the development of children's CT skills. None of the apps can ultimately support the development of CF, although ScratchJr, with a "sandbox" approach, can better help students express themselves. The author suggests that there are challenging decisions that researchers and designers have to make in relative software product creation to facilitate CT and CF for young children. Researchers also must work in this field and try innovative approaches that capitalize on helpful ideas.

Dore and Dynia investigate the use of technology and media in preschool classrooms. They go beyond prior research by surveying teachers on device use, purposes (e.g., to teach new material, entertainment), and context (individually with a teacher, as a whole class). Results show significant variation in teachers' use of these devices and several programs- and teacher-level predictors of classroom technology use.

Veraksa et al. examine the development of phonological memory in preschoolers in the context of digitalization, highlighting passive and active screen time as different influences on the processing of 
verbal information in a child. The authors demonstrate that watching television negatively predicts the development of phonological memory, while family factors such as the level of maternal education and family socio-economic status mediate this relationship.

In the fourth article, Demir-Lira et al. study the role of social robots in L2 vocabulary learning. First, the researchers have found that those children could learn L2 words from a social robot. Second, children could learn when the tutor (robot or human) gestured or used on-screen cues. Children could learn L2 words with both types of scaffolds, but learning outcomes were better when the teaching was supported by on-screen cues than when the tutor gestured. Finally, the type of gesture did not significantly influence $\mathrm{L} 2$ vocabulary learning.

In the last article, Ylinen et al. conducted a 4-week intervention focused on articulatory training and learning English words in 6-7-year-old Finnish-speaking children who used a digital language-learning game app Pop2talk. Some improvement with the Russian sibilant contrast in the gamers was not statistically significant. No improvement was observed for the tone contrast for the gaming group. Children's perceptual skills were linked to their word learning in the control group but not in the gaming group, where recurrent exposure enabled learning for children with poorer perceptual skills. Together, the results demonstrate the beneficial effects of learning via a digital application yet raise a need for further research of individual differences in learning.
The articles assembled in this volume indicate that welldesigned educational apps can improve learning outcomes. It is, therefore, necessary to enhance research on app design and the ways the latter can shape learning environments and outcomes. Longitudinal approaches may be an appropriate approach in this respect.

\section{AUTHOR CONTRIBUTIONS}

All authors listed have made a substantial, direct, and intellectual contribution to the work and approved it for publication.

Conflict of Interest: The authors declare that the research was conducted in the absence of any commercial or financial relationships that could be construed as a potential conflict of interest.

Publisher's Note: All claims expressed in this article are solely those of the authors and do not necessarily represent those of their affiliated organizations, or those of the publisher, the editors and the reviewers. Any product that may be evaluated in this article, or claim that may be made by its manufacturer, is not guaranteed or endorsed by the publisher.

Copyright (c) 2021 Papadakis, Kalogianakis, Sifaki and Monnier. This is an openaccess article distributed under the terms of the Creative Commons Attribution License (CC BY). The use, distribution or reproduction in other forums is permitted, provided the original author(s) and the copyright owner(s) are credited and that the original publication in this journal is cited, in accordance with accepted academic practice. No use, distribution or reproduction is permitted which does not comply with these terms. 